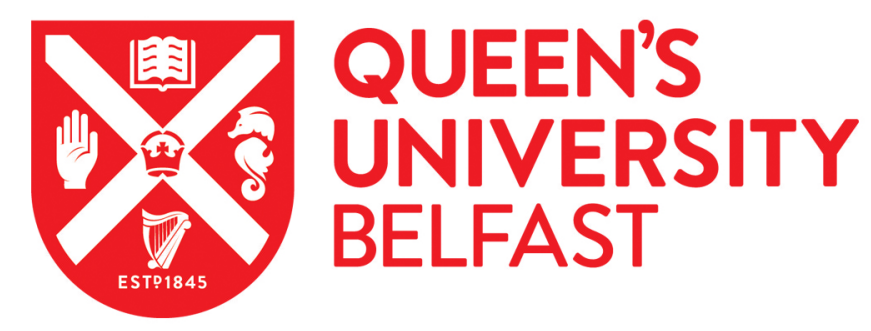

\title{
Programmable Ring Oscillator PUF Based on Switch Matrix
}

Cui, Y., Chen, Y., Gu, C., Wang, C., O'Neill, M., \& Liu, W. (2020). Programmable Ring Oscillator PUF Based on Switch Matrix. In IEEE International Symposium on Circuits and Systems 2020: Proceedings (IEEE International Symposium on Circuits and Systems (ISCAS): Proceedings). Institute of Electrical and Electronics Engineers Inc.. https://doi.org/10.1109/ISCAS45731.2020.9180552

Published in:

IEEE International Symposium on Circuits and Systems 2020: Proceedings

Document Version:

Peer reviewed version

Queen's University Belfast - Research Portal:

Link to publication record in Queen's University Belfast Research Portal

Publisher rights

(c) 2020 IEEE.

This work is made available online in accordance with the publisher's policies. Please refer to any applicable terms of use of the publisher.

\section{General rights}

Copyright for the publications made accessible via the Queen's University Belfast Research Portal is retained by the author(s) and / or other copyright owners and it is a condition of accessing these publications that users recognise and abide by the legal requirements associated with these rights.

Take down policy

The Research Portal is Queen's institutional repository that provides access to Queen's research output. Every effort has been made to ensure that content in the Research Portal does not infringe any person's rights, or applicable UK laws. If you discover content in the Research Portal that you believe breaches copyright or violates any law, please contact openaccess@qub.ac.uk. 


\title{
Programmable Ring Oscillator PUF based on Switch Matrix
}

\author{
Yijun Cui ${ }^{1}$, Yunpeng Chen ${ }^{1}$, Chenghua Wang ${ }^{1}$, Chongyan $\mathrm{Gu}^{1}$, Máire O’Neill ${ }^{2}$ and Weiqiang Liu ${ }^{1}$ \\ ${ }^{1}$ College of Electronic and Information Engineering, Nanjing University of Aeronautics and Astronautics, Nanjing, China \\ ${ }^{2}$ Centre for Secure Information Technologies, Queen's University Belfast, Belfast, UK
}

\begin{abstract}
Configurable ring oscillator (CRO) physical unclonable functions (PUFs) which can improve the uniqueness and reliability of conventional RO PUFs have been widely studied. Especially, the multiplier, XOR gate and tristate inverter based CRO PUFs can improve the uniqueness and reliability. However the efficiency is remain at the same level when compared with the conventional RO PUFs. In this paper, a programmable RO PUF (PRO PUF), which can be programmed to change the structure of a typical RO PUF, is proposed. The proposed PRO PUF design is implemented based on the switch matrix of an FPGA and can be programmed as a chained RO PUF or a random looped RO PUF. The proposed PRO PUF is implemented on Xilinx Spartan 6 FPGAs. Experimental results demonstrate that the proposed PRO PUF design has good uniqueness and reliability metrics as well as a high hardware efficiency.
\end{abstract}

Index Terms-Configurable ring oscillator PUF; Switch matrix; FPGAs

\section{INTRODUCTION}

$\mathbf{P}$ HYSICAL unclonable functions (PUFs) are widely studied and applied to the Internet of Things (IoTs). One of the most important characteristics of PUFs is that it is unclonable even by manufacturers. The challenge-response pairs (CRPs) generated by PUFs are only effected by the random noise introduced in the manufacture process, which is difficult to be predicted.

There are many different types of PUFs, such as Arbiter PUF [1], SRAM PUF [2] and ring oscillator PUF (RO PUF) [3]. The first RO PUF was proposed in [3], in which the frequency difference between two ROs is used as the output of the PUF.

RO PUF is one of the most widely studied PUF designs due to its high uniqueness and reliability. Also it can be easily implemented on various FPGAs compared with other PUFs. However, RO PUF has low hardware efficiency. For an ASIC implementation, hundreds of inverters are needed to compose one RO array. Configurable RO (CRO) PUF [4] was proposed to improve the efficiency of the conventional RO PUF. Configurable units, e.g. MUX and tristate inverters are utilized to determine the transmission path and select the inverters to compose a basic RO. Hence, more CRPs can be generated and hardware resource consumption is reduced.

The previous CRO PUF maintained the same uniqueness and reliability as the RO PUF while the hardware efficiency has not been improved significantly. In this paper, a novel PRO PUF is proposed based on the switch matrix of FPGAs. Compared with the previous CRO PUFs, the proposed PUF design can be programmed to different patterns like a random loop RO PUF by adjusting its propagation path and direction. When the proposed PRO PUF design is implemented on an FPGA, the Programmable Interconnect Points (PIPs) can be utilized to implement the switch matrix, which is very abundant in an FPGA. Compared with previous CRO PUFs, the PRO PUF can achieve a good uniqueness and reliability as well as a higher hardware efficiency. The contributions of this paper are summarized as follows:

- A PRO PUF design based on the switch matrix is proposed. The transmission direction, numbers of inverters and propagation path for the proposed PRO PUF can be programmed. To the best of the author's knowledge, this is the first PUF design based on the switch matrix.

- The efficiency and flexibility of the CRO PUF is improved. The PRO PUF can generate more CRPs, since it provides more programming or configuring combinations while consuming same hardware resources. The result shows that the proposed PRO PUF design can exponentially increase the number of CRPs.

- The proposed PRO PUF design provides high entropy. The switch matrix can transmit the signal in several directions which means the proposed PRO PUF design can be programmed to different patterns.

- The proposed PUF is implemented on Spartan 6 series FPGAs. The experimental results shows that the proposed PRO PUF design has a good uniqueness $(44.79 \%)$, reliability (98.01\%) as well as a high hardware efficiency.

The remainder of this paper is organized as follows. Section II presents the detailed design of the PRO PUF. FPGA implementation of the PRO PUF is given in Section III. Experimental details and results are presented in Section IV. Section V concludes this paper.

\section{Proposed Programmable RO PUF}

Inspired by the routing strategy for FPGAs, a novel programmable RO PUF, PRO PUF, is proposed in this paper. Compared with conventional RO PUFs and configurable RO PUFs, the new PUF design can be programmed to be different RO PUF designs to generate specific challenge and response bits.

\section{A. PRO PUF Cell Circuits}

In the proposed PUF design, the basic elements is a switch box (SB) [5]. As an example, a SB with flexibility (the number 


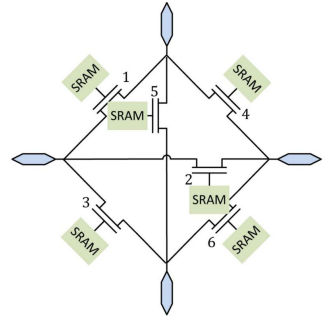

(a)

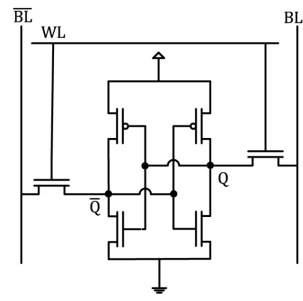

(b)
Fig. 1. SB architecture: (a) Typical SB programmed by six SRAMs. (b) Typical SRAM used as configuration memory in SBs.

of possible outputs corresponding to each input) of three is shown in Fig. 1(a). Inside the SB, the SRAM cells can be programmed to change the configuration (or pattern) of the SB. We have numbered the pass transistors in Fig. 1(a) for further references regarding SB patterns. A typical implementation of a SRAM configuration memory is shown in Fig. 1(b).

Each switch in the proposed PRO PUF cell is composed of six transmission gates and six configurable SRAMs. If the value of the SRAM is ' 0 ', the transmission gate is off or disconnected. If the value of the SRAM is ' 1 ', the transmission gate is on and connected the two points. The configure signal is denoted as $n_{i}$, where $1<i<6$, and $n_{i} \in\{0,1\}$. As shown in Fig. 1(a), according to the configurable signal, there are six connected routes in a SB.

Assuming the PRO PUF cell have a switch matrix with $x$ rows and $y$ columns. A switch can be denoted as $S(x, y)$. To configure the PUF cell, a subset of the vector,

$$
\vec{p}_{x y}=\left\{n_{1}, n_{2}, n_{3}, n_{4}, n_{5}, n_{6}\right\}
$$

is applied to $S(x, y)$ to programme the switches. When the value of the element $n_{i}$ in the vector $\vec{p}_{x y}$ is ' 1 ', it indicates that the connection mode of the switch matrix is the $i_{t h}$ type in Fig. 1(a).

If the $i_{t h}$ connection of the $S(x, y)$ in a PRO PUF cell is configured, the delay signal of $S(x, y)$ can be denoted as $\tau(x, y, i)$. When $k$ switches are programmed to compose a $\mathrm{RO}$, the output frequency of the RO is

$$
f=\frac{1}{\sum_{1}^{k} \tau(x, y, i)}
$$

Due to the fabrication variations, the delay of the routes between different configurations of the same switch and that of different switches is slightly different.

A typical PRO PUF cell is illustrated in Fig. 2. It can be seen that the PRO cell is composed of a programmable delay unit and a logic gate. The programmable delay unit is a set of SBs. In a typical RO PUF circuit, a digital signal is transmitted from a source component to a target component by routing it through a chain of intermediate components. Due to the difference of delays introduced by the wires and gates, the oscillating frequency varies differently. In the proposed PRO

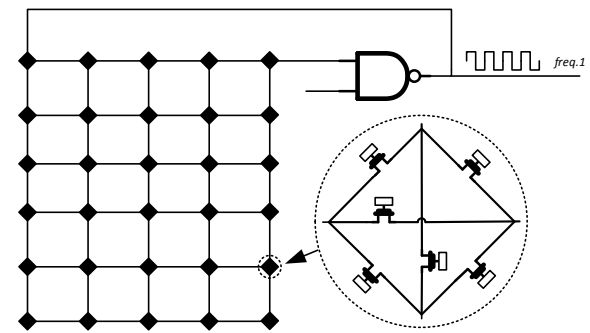

Fig. 2. PRO PUF cell circuits.

PUF, the programming signal determines the transmission of the routed signal. The delay of each component contributes to the total delay. The delay depends on wire length, capacitance, transistor switching speed, temperature and other factors.

The implementation of the proposed design only uses the switch matrix to achieve the delay. The switch matrix is the main component which can be configured to transmit a signal from one of several input pins to one (or more) of its output pins. The pins of the logic elements and the inter-tile network wires are connected to these inputs and outputs. For a given pair of input and output pins, there are many ways to route the signal within the switch matrix. This is due to the architecture of the switch matrix which can route a signal from an input pin to one of its own output pin unconnected externally, and bounce the signal to the input pin of another switch matrix. Eventually, the signal is derived from the output pin of the last matrix.

\section{B. Programming Strategies for the Proposed PRO PUF Cell}

In a CRO PUF, configurable bits should be defined. In this paper, the PRO PUF is more flexible and can be programmed to different patterns to generate more CRPs. The programming process is a procedure, determines which switch is enabled to joint the PRO PUF and which route of the switch is activated to propagate the electronic signal. For a $m \times n$ switch matrix, the programming signal is a matrix $\vec{P}$, where

$$
\vec{P}=\left(\begin{array}{cccc}
p_{11} & p_{12} & \cdots & p_{1 n} \\
p_{21} & p_{22} & \cdots & p_{2 n} \\
\cdots & \cdots & \cdots & \cdots \\
p_{m 1} & p_{m 2} & \cdots & p_{m n}
\end{array}\right)
$$

In the matrix $\vec{P}$, if the value is ' 0 ', the route of the switch is off and if the value is ' 1 ', the route of the switch is selected and activated to joint the signal propagate. Usually, there are only one route of the switch is chosen and utilized. As mentioned above, the programming vector for the illustrated example is

$$
\vec{P}=\left(\begin{array}{ll}
(000010) & (000001) \\
(000100) & (100000)
\end{array}\right)
$$

In a conventional RO PUF, the challenge will pick up a pair of ROs to compare the output frequency and generate one response bit. In order to ensure that the frequency difference between the two ROs is entirely from manufacture variations, 


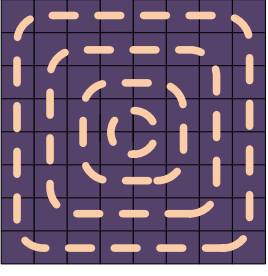

(a)

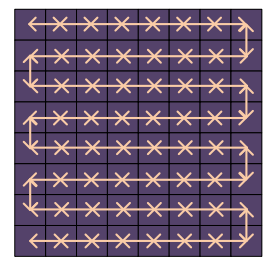

(b)
Fig. 3. (a) Loop-like programming strategy. (b) Chain-like programming strategy.

their structures must be identical. When it comes to a PRO PUF, the system have to programme the PUF cell in utilization first. The proposed PRO PUF is very flexible due to the 6direction-connection in every switch. The proposed PRO PUF cell can be programmed to different pattern depending on the users' demand. In general, to make the most efficient usage of each switch in the matrix, the proposed PRO PUF can be programmed to two different patterns, random loop and chain like, as shown in Fig. 3(a) and Fig. 3(b) respectively. The frequencies of the PRO PUF cells will be compared with each other after the programming to generate the response bits.

1) Random Loop Pattern

A random loop pattern is circle and only part of the switches are chosen, which is very easy to implement and utilized. As illustrated in Fig. 3(a), from outside to inside, there are many possible programming routes to chosen in a random loop pattern.

2) Chain Like Pattern

A chain like pattern can take full use of the switches in the matrix. The accumulation of the delays of each route and each switch make the responses bit more accurate and more stable. However, if every switch is used in the chain like pattern, the total number of the possible programming routes will decrease.

\section{FPGA IMPLEMENTATION OF THE PROPOSED PRO PUF}

To validate the proposed PRO PUF design, implementations on Xilinx Spartan 6 FPGAs are presented in this section. The significant difference between the proposed design and the existing designs is the core unit PRO cell, and the rest of the circuit is similar as the existing RO PUF designs.

In order to introduce a configuration signal (challenge) to the proposed PRO PUF, in addition to utilizing logic elements such as $X O R$ gates, dynamic reconfiguration techniques [6] can also be utilized. The dynamic reconfiguration makes it possible to partially reconfigure a part of the design while the rest is still running. In this paper, a Dynamic Xilinx Configuration Library (DXCL) proposed in [6] is adopted. In order to use dynamic reconfiguration, the first step is to divide the design into static and dynamic area. The static area is used to implement the parts of the circuit that do not need to be changed, including counters, timers, control modules, etc. The PRO cell is implemented in the dynamic area, so the

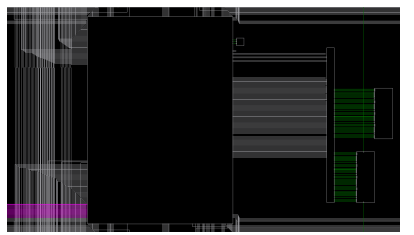

(a)

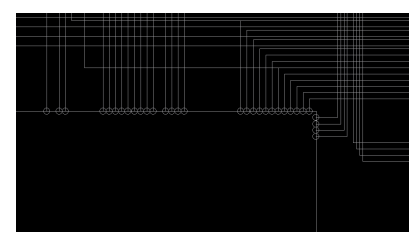

(b)
Fig. 4. (a) Switch matrix with one CLB. (b) PIPs in switch matrix.

PRO can be reconfigured during PUF operation. During the reconfiguration process, the difference between the reconfigured structure and the replaced structure is only the difference in the transmission paths of the signals in the switch matrix. The challenge signal is used to control the configuration of the PRO cell, that is, to select the transmission path in the switch matrix. Each transmission path (configuration) has a corresponding unique configuration file which is stored externally. These configuration files are numbered so that they can be indexed by challenges. For example, when the challenge $C_{i}$ is input to the system, the system will read the $C_{i t h}$ configuration file and configure the dynamic area accordingly. Since there are many possibilities for the selection of the transmission path in the switch matrix, this provides a large number of challenge bits and also greatly increases the number of CRPs.

In the implementation of the PRO PUF on the FPGA, the basic cell is composed by one switch matrix and one configurable logic block (CLB) connected. Lookup tables (LUT) in the CLB are used to implement logic functions (such as inversion, buffering). The switch matrix works as the programmable delay unit. Fig. 4(a) shows the structure of a CLB and the switch matrix connected to it. Amplifying a corner of the switch matrix is shown in Fig. 4(b). The switch matrix contains a large number of PIPs, and each circle in Fig. 4(b) represents a PIP. In order to programme these PIPs in the switch matrix, Xilinx design language (XDL) [7] is utilized.

\section{EXPERIMENTAL RESULTS}

In this section, the above-mentioned PRO PUF cell and the PRO PUF composed of it are implemented and tested. These PRO PUF cells are composed of an SB and an inverter, which can be called 1-stage PRO PUF cells.

\section{A. Ring Oscillator Outputs on FPGA}

100 instances of the proposed PRO PUF cell are implemented on a Xilinx Spartan 6 LX16 platform. The second measurement is carried out with the addition of the switch matrix delay. The results of both measurements show that the frequencies of PRO cells with the same structure still have small differences from each other. And there is no obvious rule for this difference.

\section{B. Uniqueness}

As the output response of a PUF will be used for security applications, e.g. device authentication and key generation, the responses of each chip should be unpredictable. Uniqueness 


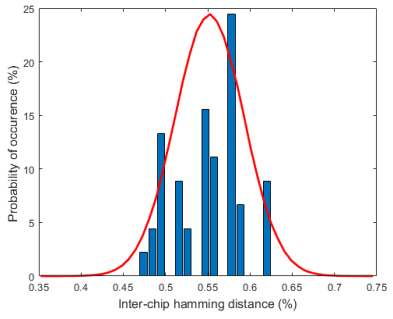

(a)

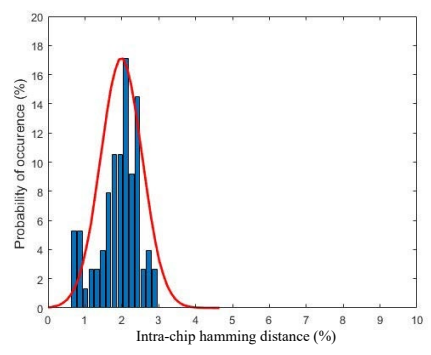

(b)
Fig. 5. (a) The uniqueness result of the proposed PRO PUF. (b) The reliability result of the proposed PRO PUF.

evaluates how easily the responses of different PUF implementations can be differentiated when the same challenge input is applied. Uniqueness can be defined according to the average inter-chip Hamming distance (HD). Ideally, the uniqueness should be $50 \%$. The uniqueness result of the PRO PUF is shown in Fig. 5(a), and the mean value is $44.79 \%$, which is close to the idle value.

\section{Reliability}

A PUF design should always produce the same response to the same challenge. However, variations in the supply voltage and temperature can affect the response. Reliability assesses the robustness of a PUF design under different environmental conditions. The bit error rate (BER) is used to measure a PUF's reliability, which can be defined by calculating the average intra-chip HD. The ideal value for reliability is $100 \%$. Fig. 5(b) is the reliability result of PRO PUF. The average bit error rate is $1.99 \%$, so the average reliability is $98.01 \%$.

\section{Hardware Efficiency}

PUF is usually used for resource-constrained platforms. Therefore, hardware efficiency (HE) is an important metric. HE can be defined as the ratio of the number of CLBs used to the number of CRP bits. There are at least 300 PIPs in a switch matrix. Assume the random loop pattern programming strategy is adopted, and only use one PIP on each of the four sides. HE can be represented as follows:

$$
H E=\frac{N_{C L B}}{N_{b i t s}}=\frac{2 m}{m(m-1) \cdot 75^{4}}
$$

where $m$ is the number of PRO cells. Fig. 6 shows the comparison of the HEs of the PRO PUF and other works. It can be seen that the HE of PRO PUF is at least $10^{5}$ times higher than the existing structures.

\section{CONClusion}

In this paper, a novel and efficient PRO PUF design is proposed. The PRO PUF is composed of a programmable switch matrix and the oscillating circuits are utilized to extract the delay difference of the switch matrix. The proposed PUF design is flexible and can generate more CRPs than conventional RO PUFs and CRO PUFs. The PRO PUF is

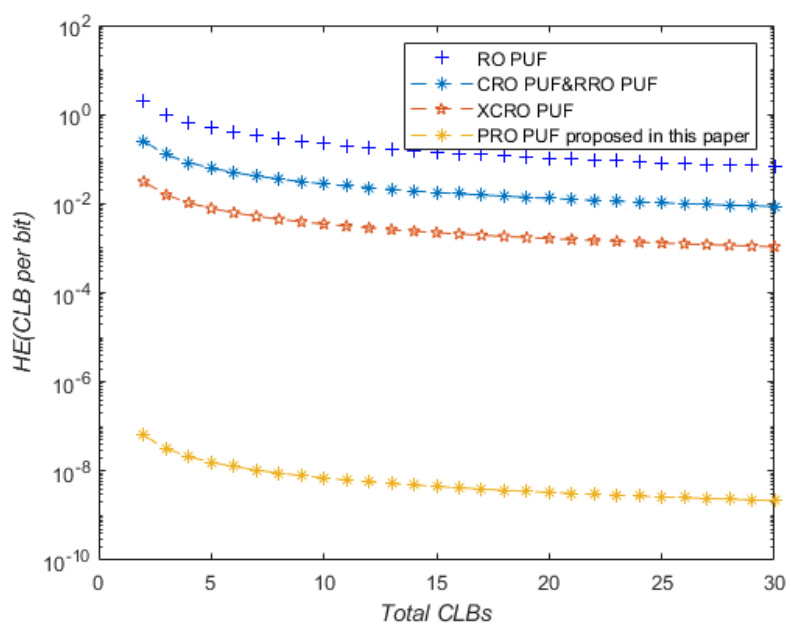

Fig. 6. Comparison of hardware efficiency with PRO PUF and RO [3], CRO [4], RRO [9], XCRO [8].

implemented on Xilinx Spartan 6 FPGAs and the experimental results show that the proposed PRO PUF design has good uniqueness and reliability results as well as a very high hardware efficiency.

\section{REFERENCES}

[1] D. Lim, J. W. Lee, B. Gassend, G. E. Suh, M. V. Dijk, and S. Devadas, "Extracting secret keys from integrated circuits," IEEE Transactions on Very Large Scale Integration Systems, vol. 13, no. 10, pp. 1200-1205, 2005.

[2] J. Guajardo, S. S. Kumar, G.-J. Schrijen, and P. Tuyls, "FPGA intrinsic PUFs and their use for IP protection," in Proc. International Workshop on Cryptographic Hardware and Embedded Systems, 2007, pp. 63-80.

[3] G. E. Suh and S. Devadas, "Physical unclonable functions for device authentication and secret key generation," in Proc. 44th ACM Annual Design Automation Conference, 2007, pp. 9-14.

[4] A. Maiti and P. Schaumont, "Improving the quality of a physical unclonable function using configurable ring oscillators," in Proc. IEEE International Conference on Field Programmable Logic and Applications(FPL), 2009, pp. 703-707.

[5] G.-M. Wu, M. Shyu, and Y.-W. Chang, "Universal switch blocks for three-dimensional FPGA design," IEE Proceedings-Circuits, Devices and Systems, vol. 151, no. 1, pp. 49-57, 2004.

[6] B. Blodget, C. Bobda, M. Hübner, and A. Niyonkuru, "Partial and dynamically reconfiguration of Xilinx Virtex-II FPGAs," in Proc. International Conference on Field Programmable Logic and Applications, 2004, pp. 801-810.

[7] C. Beckhoff, D. Koch, and J. Torresen, "The Xilinx Design Language (XDL): Tutorial and use cases," in Proc. 6th International Workshop on Reconfigurable Communication-Centric Systems-on-Chip (ReCoSoC), 2011, pp. 1-8.

[8] L. Zhang, C. Wang, W. Liu, M. O'Neill, and F. Lombardi, "XOR gate based low-cost configurable RO PUF," in Proc. IEEE International Symposium on Circuits and Systems (ISCAS), 2017, pp. 1-4.

[9] Y. Cui, C. Wang, W. Liu, Y. Yu, M. O'Neill, and F. Lombardi, "Lowcost configurable ring oscillator PUF with improved uniqueness," in Proc. IEEE International Symposium on Circuits and Systems (ISCAS), 2016, pp. 558-561. 